\title{
Espectro e distribuição vertical das estratégias de dispersão de diásporos do componente arbóreo em uma floresta estacional no sul do Brasil
}

\author{
Eduardo Luís Hettwer Giehl ${ }^{1,4}$, Eduardo Anversa Athayde², Jean Carlos Budke ${ }^{1}$, \\ João Pedro Arzivenko Gesing ${ }^{3}$, Sônia Maria Einsiger ${ }^{3}$ e Thais Scotti do Canto-Dorow ${ }^{3}$
}

Recebido em 13/04/2006. Aceito em 17/08/2006

\begin{abstract}
RESUMO - (Espectro e distribuição vertical das estratégias de dispersão de diásporos do componente arbóreo em uma floresta estacional no sul do Brasil). A dispersão de diásporos é um evento de elevada importância para as espécies vegetais, que dispõem de diversas estratégias para este fim. O presente estudo teve por objetivos conhecer e caracterizar o espectro das diferentes estratégias de dispersão do componente arbóreo em uma floresta estacional de Santa Maria, Rio Grande do Sul, Brasil (próximo às coordenadas $53^{\circ} 54^{\prime} \mathrm{W}$ e $2^{\circ} 38^{\prime} \mathrm{S}$ ) e, a partir desses dados, investigar sua relação com os estratos verticais da floresta. Foram amostrados todos os indivíduos com perímetro à altura do peito $\geq 15 \mathrm{~cm}$, em 100 unidades amostrais de $10 \times 10 \mathrm{~m}$. A caracterização das estratégias de dispersão foi realizada por meio de observações a campo de frutos e potenciais dispersores, e consulta à bibliografia especializada. Foram amostradas 58 espécies pertencentes a 26 famílias botânicas. Dessas espécies, 74\% apresentaram estratégia de dispersão zoocórica; $24 \%$, estratégia de dispersão anemocórica e apenas Gymnanthes concolor Spreng. apresentou a estratégia autocórica. Analisando-se a proporção das estratégias de dispersão por meio da densidade relativa, verificou-se que $80 \%$ dos indivíduos são zoocóricos, $12 \%$ anemocóricos e $8 \%$ autocóricos. Para a floresta analisada foram encontradas diferenças significativas nos padrões de distribuição vertical das espécies agrupadas de acordo com a estratégia de dispersão. A zoocoria ocorreu em espécies de todos os estratos, enquanto a autocoria ficou limitada ao sub-bosque. Já a anemocoria foi mais importante entre as espécies com indivíduos emergentes.
\end{abstract}

Palavras-chave: estratégias de dispersão, estrutura vertical, dispersão de sementes, zoocoria, espectro de dispersão

\begin{abstract}
Spectrum and vertical distribution of diaspore dispersal modes in a seasonal forest in Southern Brazil). Diaspore dispersal is presumed to have great fitness for plants, which could be enhanced by different dispersal modes. Our aim was to generate a dispersal spectrum and to seek for analyze relative ecological importance of tree-species dispersal modes in a seasonal forest in Santa

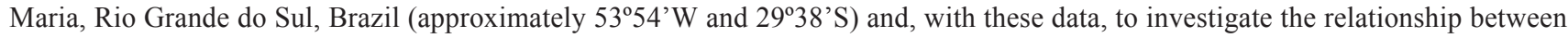
vertical structure of the forest and dispersal strategies. The tree survey included all individuals with perimeter at breast height $\geq 15 \mathrm{~cm}$, in 100 sample units $(10 \times 10 \mathrm{~m})$. We classified dispersal modes by diaspores and potential dispersers in the field, and when necessary through specialized bibliography. Fifty-eight tree species from 26 families were sampled. Of these species, 74\% are zoochoric, 24\% anemochoric and only Gymnanthes concolor Spreng. is autochoric. By analyzing the proportion of dispersal strategies based on relative density, we found that $80 \%$ of the trees are zoochoric, $12 \%$ anemochoric and $8 \%$ autochoric. In the study area, we found significant differences in vertical distribution patterns of species grouped by dispersal strategies. Zoochory occurred in all vertical strata while autochory was limited to the understorey. Anemochory occurred mainly in species with emergent trees.
\end{abstract}

Key words: dispersal modes, dispersal spectrum, vertical structure, seed dispersal, zoochory

\section{Introdução}

Um dos eventos de importância vital para as espécies vegetais é o afastamento de sementes ou frutos inteiros da planta-mãe, processo chamado de dispersão (Howe \& Smallwood 1982; Howe 1986). Esse processo permite que as espécies ampliem suas áreas de ocorrência, tenham um incremento nas taxas de sobrevivência dos indivíduos jovens, seja pela redução da competição intra-específica ou pela diminuição da quantidade e concentração de predadores (Janzen 1980), e possibilita flutuações genéticas entre populações (van der Pij1 1982).

A dispersão também modifica os padrões de recrutamento em escala espacial e temporal. Para espécies dependentes de condições especiais para o estabelecimento das plântulas, como clareiras com ocorrência espacial e temporal variável no ambiente

\footnotetext{
1 Universidade Federal do Rio Grande do Sul, Programa de Pós-Graduação em Botânica, Av. Bento Gonçalves 9500, Prédio 43433, $91105-970$ Porto Alegre, RS, Brasil

2 Universidade Estadual Paulista, Programa de Pós-Graduação em Biologia Vegetal, Av. 24A, 1515, 13506-900 Rio Claro, SP, Brasil

3 Universidade Federal de Santa Maria, Departamento de Biologia, Av. Roraima 1000, Prédio 16, 97105-900 Santa Maria, RS, Brasil

4 Autor para correspondência: eduardohet@gmail.com
} 
florestal (Augspurger 1983), o transporte dos diásporos é essencial para a colonização de novos sítios (Hamilton \& May 1977). Desta forma, a estrutura e a dinâmica das comunidades vegetais é influenciada pela eficiência da dispersão (Sinha \& Davidar 1992).

Existem várias estruturas morfológicas que facilitam a dispersão dos diásporos (Hughes et al. 1994). De acordo com van der Pijl (1982), os diásporos podem ser classificados em três categorias: anemocóricos, quando o diásporo apresenta estruturas para facilitar o transporte pelo vento; autocóricos, quando a planta dispõe de mecanismos próprios para o lançamento de frutos e/ou sementes; e zoocóricos, quando a dispersão é propiciada pelo transporte dos frutos por animais.

A proporção relativa das estratégias utilizadas pelas espécies de um determinado tipo vegetacional é conhecida como espectro de dispersão (Hughes et al. 1994). Na composição florística da maioria das florestas tropicais e subtropicais existe maior proporção de espécies arbóreas com diásporos propensos à dispersão zoocórica, seguidas por anemocóricas e autocóricas (Costa et al. 1992; Foster et al. 1986; Howe \& Smallwood 1982; Morellato 1995; Spina et al. 2001; Willson et al. 1989).

Nas florestas estacionais do Rio Grande do Sul, estudos sobre o espectro de dispersão, embora não muito numerosos, já foram realizados por Nascimento et al. (2000), Alberti (2000) e Budke et al. (2005). Nestas, predomina de modo absoluto a zoocoria e existe uma maior equivalência entre anemocoria e autocoria, devido principalmente à presença de um grande número de espécies com frutos explosivos da família Euphorbiaceae. Neste tipo vegetacional predominam características peculiares, tais como um dossel descontínuo, com poucas árvores emergentes e geralmente três estratos adicionais (dossel, subdossel e sub-bosque) de difícil distinção em certos trechos.

Quanto à estrutura vertical, Hughes et al. (1994) propuseram alguns padrões, como uma limitação de altura para espécies autocóricas e, por outro lado, a ausência de ventos, dificultando a dispersão anemocórica no interior de florestas. Os mesmos autores assinalaram ainda a baixa ocorrência de epizoocoria (caracterizada pela presença de ganchos ou estruturas adesivas) em plantas com mais de dois metros de altura, para as quais existiriam poucos dispersores viáveis atualmente.

Os objetivos do presente estudo foram descrever e avaliar o espectro de dispersão para um fragmento de floresta estacional e, concomitantemente, tentar responder as seguintes questões: que alterações ocorrem no espectro de dispersão quando este é avaliado pelas densidades relativas das espécies? Existe diferenciação na distribuição vertical das espécies de acordo com a estratégia de dispersão adotada?

\section{Material e métodos}

Caracterização da área de estudo - A área de estudo localiza-se no distrito de Boca do Monte, município de Santa Maria, RS, com ponto central da amostra próximo às coordenadas $53^{\circ} 54^{\prime} \mathrm{W}$ e $29^{\circ} 38^{\prime} \mathrm{S}$. O fragmento florestal é de propriedade particular, com cerca de 60 ha de área total, situado numa encosta de exposição nordeste, com altitudes que variam, aproximadamente, de 200 a 300 m.s.m. Ao contrário dos fragmentos adjacentes, o trecho parece não ter sofrido ação antrópica intensa recentemente, havendo apenas registros de retirada seletiva de algumas espécies madeiráveis nas áreas de borda. A amostra foi alocada no núcleo do fragmento, onde não foram detectados distúrbios antrópicos recentes.

Segundo Teixeira et al. (1986), a referida formação florestal é denominada como pertencente à Floresta Estacional Decidual, a qual estende-se pelas encostas da formação Serra Geral, alcançando as áreas planas da depressão central, sobretudo pelas calhas dos rios Jacuí e Ibicuí. A deciduidade da floresta é atribuída principalmente à presença de um grande número de espécies da família Fabaceae (Leite 2002), que perdem as folhas no inverno devido às baixas temperaturas. Em Santa Maria, a flora das encostas é relativamente homogênea, existindo maior alteração de abundância de espécies no sub-bosque e dossel do que entre as espécies emergentes predominantemente decíduas de um local a outro.

$\mathrm{O}$ clima da região é do tipo $\mathrm{C} f a$, segundo a classificação de Köppen (Moreno 1961), apresentando chuvas bem distribuídas durante o ano e temperatura média anual superior a $18^{\circ} \mathrm{C}$ (detalhes em Budke et al. 2004a). Os solos da área são classificados como Chernossolo Argilúvico férrico típico em associação com Neossolo Litólico Eutrófico típico (Streck et al. 2002), com a presença típica de afloramentos rochosos basálticos, freqüentes nas escarpas da Serra Geral.

Levantamento e classificação dos diásporos - A amostra consistiu de 100 parcelas quadradas (MuellerDombois \& Ellenberg 1974), de 10×10 m de dimensão, dispostas em uma grade contígua de $10 \times 10$ parcelas, totalizando 1 ha de área amostrada. Todos os indivíduos 
arbóreos com perímetro à altura do peito (PAP) $\geq 15 \mathrm{~cm}$ foram amostrados e tiveram suas alturas estimadas por comparação a um cano de PVC graduado de $10 \mathrm{~m}$ de altura. Os perímetros registrados foram convertidos em diâmetros.

O material fértil coletado foi etiquetado, identificado e herborizado no Laboratório de Sistemática Vegetal (LABSIS), na Universidade Federal de Santa Maria (UFSM), sendo depositado posteriormente no herbário SMDB. As espécies foram agrupadas nas famílias reconhecidas por Tryon \& Tryon (1982) para Pterydophyta - devido à presença de Alsophila setosa, pteridófita arborescente, e pelo "Angiosperm Phylogeny Group" (APG II 2003), para Magnoliophyta.

Adotou-se a classificação de diásporos proposta por van der Pijl (1982). Para isto, durante o trabalho de campo, que transcorreu de maio/2003 a dezembro/2004, foram realizadas observações diretas dos padrões morfológicos de frutos e sementes, e, principalmente, para as espécies cuja frutificação não foi constatada, foram efetuadas consultas à bibliografia especializada (van der Pijl 1957; Janson 1983; Oliveira \& Moreira 1992; Tabarelli 1992; Barroso et al. 1999). Os diásporos foram classificados como anemocóricos quando apresentaram alas, plumas ou quando eram pequenos o suficiente para serem carregados pelo vento; zoocóricos quando apresentaram atrativos e/ou fontes alimentares a animais, e; autocóricos quando a dispersão ocorria pela explosão do fruto, também chamada de dispersão balística. Não ocorreram espécies com ganchos ou estruturas adesivas peculiares à dispersão epizoocórica em seus diásporos. Todos os indivíduos da amostra foram classificados conforme a estratégia típica da espécie.

Análise dos dados - Foram calculadas as proporções de espécies e indivíduos para cada uma das três estratégias de dispersão encontradas. Os indivíduos foram classificados de acordo com a altura em classes com amplitudes variáveis, aproximadas à extensão dos estratos da floresta. Assim, a primeira classe abrangeu indivíduos com no máximo $8 \mathrm{~m}$, valor considerado limite de altura para as espécies do sub-bosque; a segunda, indivíduos com no máximo $12 \mathrm{~m}$, altura máxima do subdossel e mínima aproximada do dossel; a terceira, com limite superior em $16 \mathrm{~m}$, correspondente ao final do dossel; e a quarta, indivíduos com altura superior a $16 \mathrm{~m}$, emergentes. Para cada classe foi analisada a freqüência relativa de cada estratégia de dispersão.

A média das alturas dos indivíduos anemocóricos, autocóricos e zoocóricos foi avaliada por análise de variância (ANOVA) em postos de Kruskal-Wallis (dados não-normalizados), ao nível de significância de $5 \%$, e comparada em grupos pareados pelo método de Dunn devido ao diferente número de amostras em cada grupo (Callegari-Jacques 2003). Para o par anemocoria-zoocoria foram efetuadas comparações adicionais, por meio do teste- $t$ de Student, para as médias das alturas máximas alcançadas pelas espécies em seus respectivos grupos de dispersão, após a verificação da normalidade dos dados (CallegariJacques 2003). Essa comparação foi utilizada para reduzir a influência de indivíduos jovens, não reprodutivos, nas conclusões sobre a estratificação.

\section{Resultados e discussão}

Espectro de dispersão - Das 58 espécies amostradas, distribuídas em 49 gêneros e 26 famílias botânicas, 43 possuem estratégia de dispersão zoocórica (74\%), 14 são anemocóricas (24\%) e apenas Gymnanthes concolor é autocórica. A lista de espécies, famílias, estratégia de dispersão e densidade absoluta é apresentada na Tab. 1.

Esse espectro assemelha-se ao encontrado por Alberti et al. (2000) e Budke et al. (2005) em Santa Maria, e é diferente do encontrado por Nascimento et al. (2000) em uma área de floresta estacional distante $110 \mathrm{~km}$ do município (Tab. 2), embora a existência de variações nos critérios de inclusão impeça generalizações precisas. Por exemplo, os padrões encontrados por Budke et al. (2005) são bastante semelhantes aos do presente estudo para a proporção de espécies anemocóricas devido à inclusão de lianas no primeiro e não devido às espécies arbóreas emergentes, que são menos diversificadas nas florestas às margens de rios na região. Já as diferenças dos trabalhos de Santa Maria em relação ao trabalho de Nascimento et al. (2000) foram atribuídas ao incremento progressivo de espécies do corredor atlântico em direção ao leste do estado. De qualquer forma, diferenças locais no espectro de dispersão são esperadas em virtude de variações na composição da flora. Estas podem ser atribuídas às características de cada sítio, devido às suas peculiaridades edáficas e topográficas e ao estádio de sucessão, além dos níveis de intervenção antrópica direta (extração seletiva de espécies e dispersão de espécies exóticas) e indireta (extinção de animais dispersores). Como grande parte destes parâmetros tem efeitos que não foram mensurados, é impossível traçar com maior detalhamento a origem das diferenças encontradas. 
Tabela 1. Listagem das espécies com estratégia de dispersão (ED) anemocórica (An), autocórica (Au) ou zoocórica (Z), densidade absoluta (DA) e voucher $(V)$, amostradas em uma floresta estacional no município de Santa Maria, RS, Brasil. Coletores: Eduardo Luís Hettwer Giehl (ELHG) e Jean Carlos Budke (JCB)

\begin{tabular}{|c|c|c|c|}
\hline Família/Espécie & ED & $\mathrm{DA}$ & $V$ \\
\hline \multicolumn{4}{|l|}{ ANNONACEAE } \\
\hline Rollinia salicifolia Schltdl. & Z & 13 & JCB 324 \\
\hline \multicolumn{4}{|l|}{ ARALIACEAE } \\
\hline Schefflera morototoni (Aubl.) Maguire, Steyerm. \& Frodin & $\mathrm{Z}$ & 39 & - \\
\hline \multicolumn{4}{|l|}{ ARECACEAE } \\
\hline Syagrus romanzoffiana (Cham.) Glass. & Z & 1 & - \\
\hline \multicolumn{4}{|l|}{ BIGNONIACEAE } \\
\hline Jacaranda micrantha Cham. & An & 3 & - \\
\hline Tabebuia heptaphylla (Vell.) Toledo & An & 4 & - \\
\hline \multicolumn{4}{|l|}{ BORAGINACEAE } \\
\hline Cordia americana L. & An & 11 & - \\
\hline C. ecalyculata Vell. & Z & 3 & - \\
\hline C. trichotoma (Vell.) Arráb. ex Steud. & An & 4 & - \\
\hline \multicolumn{4}{|l|}{ CANNABACEAE } \\
\hline Trema micrantha (L.) Blume & $\mathrm{Z}$ & 1 & - \\
\hline \multicolumn{4}{|l|}{ CARDIOPTERIDACEAE } \\
\hline Citronella paniculata (Mart.) R.A. Howard & $\mathrm{Z}$ & 9 & - \\
\hline \multicolumn{4}{|l|}{ CELASTRACEAE } \\
\hline Maytenus muelleri Schwacke & $\mathrm{Z}$ & 1 & - \\
\hline \multicolumn{4}{|l|}{ CYATHEACEAE } \\
\hline Alsophila setosa Kaulf. & An & 60 & ELHG 38 \\
\hline \multicolumn{4}{|l|}{ EBENACEAE } \\
\hline Diospyros inconstans Jacq. & $\mathrm{Z}$ & 39 & JCB 359 \\
\hline \multicolumn{4}{|l|}{ EUPHORBIACEAE } \\
\hline Alchornea triplinervea (Spreng.) Müll. Arg. & Z & 8 & ELHG 28 \\
\hline Gymnanthes concolor (Spreng.) Müll. Arg. & $\mathrm{Au}$ & 116 & JCB 301 \\
\hline \multicolumn{4}{|l|}{ FABACEAE } \\
\hline Albizia niopoides (Spruce ex Benth.) Burkart & An & 5 & ELHG 13 \\
\hline Apuleia leiocarpa (Vogel) J.F. Macbr. & An & 13 & ELHG 16 \\
\hline Enterolobium contortisiliquum (Vell.) Morong & $\mathrm{Z}$ & 9 & ELHG 25 \\
\hline Erythrina falcata Benth. & An & 3 & - \\
\hline Inga marginata Willd. & $\mathrm{Z}$ & 18 & - \\
\hline Machaerium paraguariense Hassl. & An & 4 & ELHG 22 \\
\hline Parapiptadenia rigida (Benth.) Brenan & An & 2 & ELHG 18 \\
\hline \multicolumn{4}{|l|}{ LAURACEAE } \\
\hline Aiouea saligna Meisn. & Z & 166 & ELHG 17 \\
\hline Nectandra lanceolata Nees & $\mathrm{Z}$ & 11 & ELHG 33 \\
\hline N. megapotamica (Spreng.) Mez & $\mathrm{Z}$ & 20 & - \\
\hline Ocotea puberula (Rich.) Nees & $\mathrm{Z}$ & 5 & - \\
\hline O. pulchella (Nees) Mez & $\mathrm{Z}$ & 3 & ELHG 34 \\
\hline \multicolumn{4}{|l|}{ MALVACEAE } \\
\hline Luehea divaricata Mart. \& Zucc. & An & 33 & ELHG 19 \\
\hline \multicolumn{4}{|l|}{ MELIACEAE } \\
\hline Cabralea canjerana (Vell.) Mart. & Z & 276 & ELHG 14 \\
\hline Cedrela fissilis Vell. & An & 15 & ELHG 31 \\
\hline Guarea macrophylla Vahl & $\mathrm{Z}$ & 70 & - \\
\hline Trichilia claussenii C. DC. & $\mathrm{Z}$ & 35 & ELHG 30 \\
\hline T. elegans A. Juss. & $\mathrm{Z}$ & 69 & - \\
\hline \multicolumn{4}{|l|}{ MORACEAE } \\
\hline Maclura tinctoria (L.) Don ex Steud. & $\mathrm{Z}$ & 1 & - \\
\hline Sorocea bonplandii (Baill.) W.C. Burger, Lanj. \& Boer & $\mathrm{Z}$ & 44 & - \\
\hline \multicolumn{4}{|l|}{ MYRSINACEAE } \\
\hline Myrsine umbellata Mart. & $\mathrm{Z}$ & 7 & $\begin{array}{l}\text { contir } \\
\text { con }\end{array}$ \\
\hline
\end{tabular}


Tabela 1 (continuação)

\begin{tabular}{|c|c|c|c|}
\hline Família/Espécie & ED & DA & $V$ \\
\hline \multicolumn{4}{|l|}{ MYRTACEAE } \\
\hline Campomanesia xanthocarpa $\mathrm{O}$. Berg & $\mathrm{Z}$ & 16 & JCB 181 \\
\hline Eugenia involucrata DC. & $\mathrm{Z}$ & 12 & JCB 176 \\
\hline E. ramboi D. Legrand & $\mathrm{Z}$ & 2 & JCB 240 \\
\hline E. rostrifolia D. Legrand & $\mathrm{Z}$ & 1 & - \\
\hline Plinia rivularis (Cambess.) Rotman & Z & 3 & JCB 320 \\
\hline \multicolumn{4}{|l|}{ NYCTAGINACEAE } \\
\hline Pisonia zapallo Griseb. & $\mathrm{Z}$ & 8 & ELHG 32 \\
\hline \multicolumn{4}{|l|}{ ROSACEAE } \\
\hline Prunus myrtifolia (L.) Urb. & $\mathrm{Z}$ & 24 & - \\
\hline \multicolumn{4}{|l|}{ RUBIACEAE } \\
\hline Chomelia obtusa Cham. \& Schltdl. & Z & 10 & ELHG 35 \\
\hline Randia ferox (Cham. \& Schltdl.) DC. & $\mathrm{Z}$ & 4 & JCB 245 \\
\hline \multicolumn{4}{|l|}{ RUTACEAE } \\
\hline Hellieta apiculata Benth. & An & 8 & ELHG 15 \\
\hline Zanthoxylum petiolare A. St.-Hill. \& Tul. & $\mathrm{Z}$ & 1 & ELHG 36 \\
\hline Z. rhoifolium Lam. & $\mathrm{Z}$ & 1 & - \\
\hline \multicolumn{4}{|l|}{ SALICACEAE } \\
\hline Banara tomentosa Clos & $\mathrm{Z}$ & 6 & ELHG 12 \\
\hline Casearia decandra Jacq. & $\mathrm{Z}$ & 5 & JCB 277 \\
\hline C. silvestris $\mathrm{Sw}$. & $\mathrm{Z}$ & 18 & ELHG 23 \\
\hline \multicolumn{4}{|l|}{ SAPINDACEAE } \\
\hline Allophylus edulis (A.St.-Hil., Cambess. \& A. Juss.) Radlk. & $\mathrm{Z}$ & 17 & JCB 253 \\
\hline Cupania vernalis Cambess. & $\mathrm{Z}$ & 149 & - \\
\hline Matayba elaeagnoides Radlk. & Z & 8 & JCB 371 \\
\hline \multicolumn{4}{|l|}{ SAPOTACEAE } \\
\hline Chrysophyllum gonocarpum (Mart. \& Eichler) Engl. & $\mathrm{Z}$ & 8 & - \\
\hline C. marginatum (Hook. \& Arn.) Radlk. & Z & 1 & JCB 390 \\
\hline \multicolumn{4}{|l|}{ URTICACEAE } \\
\hline Bohemeria caudata $\mathrm{Sw}$. & An & 1 & ELHG 37 \\
\hline Urera baccifera (L.) Gaudich. & $\mathrm{Z}$ & 3 & - \\
\hline
\end{tabular}

Além de representar a maior proporção relativa de espécies, a zoocoria abrangeu a maioria absoluta de indivíduos (1.154 de 1.438 indivíduos no total, ou $80 \%$ ). A anemocoria foi apresentada por espécies com baixa densidade na área, principalmente da família Fabaceae, totalizando apenas 168 indivíduos (12\%). G. concolor, ao contrário, é uma espécie de alta densidade absoluta na área (Tab. 1), elevando a proporção da estratégia de dispersão autocórica (8\%) na comunidade.

Essa alteração de padrão nas porcentagens, quando incorporados os valores de densidade das espécies, já havia sido constatado por Foster et al. (1986) e Budke et al. (2005). É extremamente relevante avaliar este aspecto, visto que permite estimar a disponibilidade de recursos alimentares para animais frugívoros (Willson et al. 1989). Budke et al. (2005) constataram redução da contribuição da estratégia zoocórica, resultado contrário ao do presente estudo. A elevada densidade relativa da estratégia autocórica na floresta ribeirinha analisada por Budke et al. (2005) parece ocorrer devido à limitação imposta pelas inundações periódicas, favorecendo espécies tolerantes autocóricas da família Euphorbiaceae (Budke et al. 2004b).

Distribuição vertical - A distribuição dos indivíduos em classes de altura (Fig. 1), denotou uma dominância numérica de árvores de pequeno porte. A primeira classe foi representada por G. concolor (autocórica), Alsophila setosa (anemocórica) e várias espécies zoocóricas, como Guarea macrophylla, Trichilia elegans e Sorocea bonplandii. Na segunda classe, com freqüências relativamente maiores do que nas demais, ocorreram Aiouea saligna, Chomelia obtusa e Trichilia clausseni (zoocóricas). Cabralea canjerana, Cupania vernalis, Diospyros inconstans, Inga marginata e Prunus myrtifolia (zoocóricas), Cordia americana e Luehea divaricata (anemocóricas), ocorreram freqüentemente até a 
Tabela 2. Proporções de espécies e de abundância de indivíduos anemocóricos (An), autocóricos (Au), e zoocóricos (Z) de florestas estacionais no município de Santa Maria, RS, Brasil.

\begin{tabular}{|c|c|c|c|c|c|c|c|}
\hline & \multirow[b]{2}{*}{ Tipo florestal } & \multicolumn{3}{|c|}{ Espécies (\%) } & \multicolumn{3}{|c|}{ Abundância (\%) } \\
\hline & & An & $\mathrm{Au}$ & $\mathrm{Z}$ & An & $\mathrm{Au}$ & $\mathrm{Z}$ \\
\hline Presente estudo & Floresta estacional & 24 & 2 & 74 & 12 & 8 & 80 \\
\hline Nascimento et al. (2000) & Floresta estacional & 9 & 13 & 78 & - & - & - \\
\hline Alberti et al. (2000) & Floresta estacional & 23 & 12 & 65 & - & - & - \\
\hline Budke et al. (2005) & Floresta estacional ribeirinha & 24 & 4 & 72 & 43 & 12 & 45 \\
\hline
\end{tabular}

terceira classe. A quarta classe foi representada principalmente por Apuleia leiocarpa e Cedrela fissilis, além de outras fabáceas anemocóricas, e por Schefflera morototoni, Nectandra lanceolata e N. megapotamica (zoocóricas). A Fig. 2 permite visualizar a ausência de indivíduos autocóricos a partir da terceira classe e um incremento da proporção de indivíduos anemocóricos nas classes de alturas mais elevadas. Também é possível observar a predominância de indivíduos zoocóricos na primeira classe de altura, correspondente ao sub-bosque, que devido ao grande número de indivíduos, potencialmente dispõem de grande parte dos recursos disputados pelos animais frugívoros da floresta.

A comparação entre grupos de dispersão, por meio de análise de variância, indicou diferenças significativas quando comparadas as alturas dos indivíduos autocóricos com as dos zoocóricos $(\mathrm{P}<0,05)$ e dos autocóricos com as dos anemocóricos $(\mathrm{P}<0,05)$. Sob este teste, não existiram diferenças significativas entre as alturas dos indivíduos zoocóricos e anemocóricos. Essas diferenças sugerem uma utilização distinta dos espaços verticais da floresta pela autocoria, que ocorre apenas nas menores classes de altura. Esta

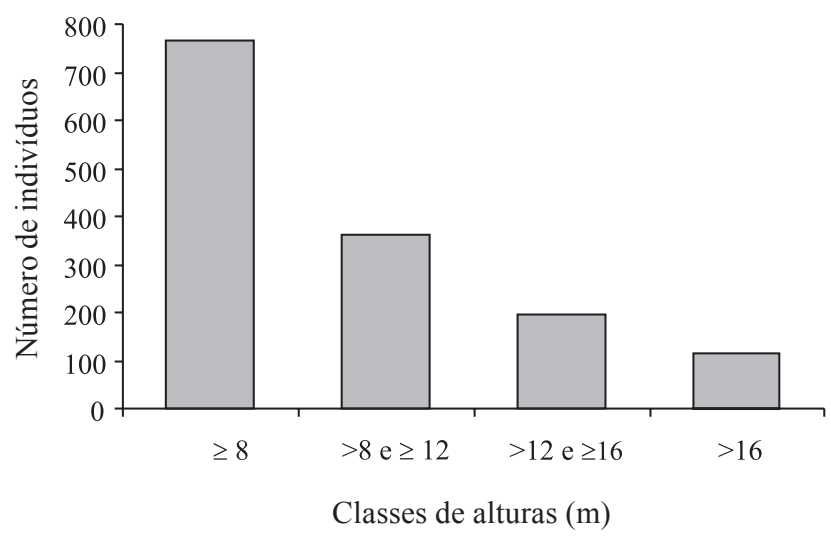

Figura 1. Distribuição dos indivíduos arbóreos em classes de altura de uma floresta estacional no município de Santa Maria, RS, Brasil.

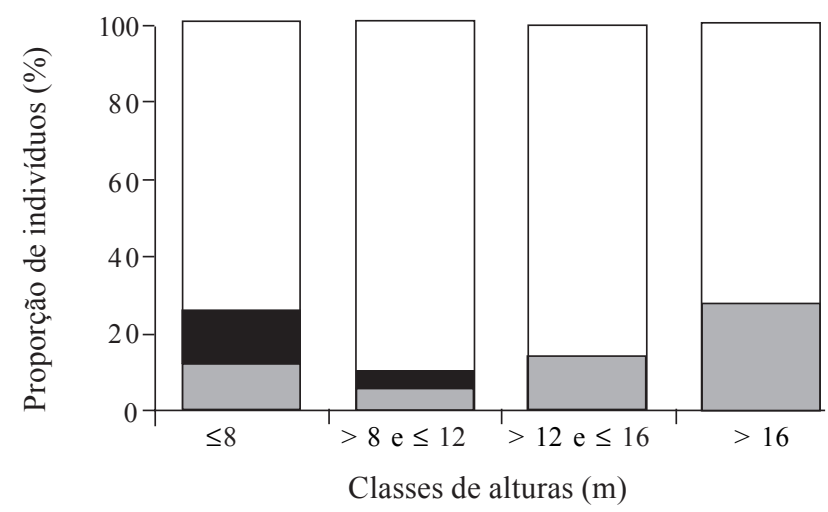

Figura 2. Distribuição relativa das diferentes estratégias de dispersão, de acordo com as classes de altura de floresta estacional no município de Santa Maria, RS, Brasil. ( $\square=$ Zoocoria;

$\mathbf{\square}=$ Autocoria; $\mathbf{n}=$ Anemocoria).

constatação é condizente com a revisão de Hughes et al. (1994), na qual concluíram que o escape efetivo da competição intra-específica por meio de mecanismos de dispersão balísticos só pode ser alcançado se a árvore mãe possuir pequenas dimensões. Esse efeito é inerente à própria limitação imposta pelo mecanismo físico da propulsão balística, que impede o alcance de longas distâncias pelas sementes (Willson 1993).

Foi constatado que Gymnanthes concolor, única espécie autocórica amostrada, apresentou um padrão de distribuição espacial agrupado, possivelmente refletindo uma limitação de seu mecanismo de dispersão, efetivo apenas para o deslocamento dos diásporos a curtas distâncias. A espécie suporta elevadas densidades (por exemplo: 632 ind. ha ${ }^{-1} \mathrm{em}$ Jarenkow \& Waechter 2001; 715 ind. ha ${ }^{-1}$ em Budke et al. 2004b), por ser esciófila e de pequeno porte (Marchiori 2000), e também, possivelmente, devido à tolerância à competição intra-específica e a um alto poder competitivo interespecífico com outras plântulas durante o estabelecimento inicial. Estas características, associadas à distribuição de sementes apenas para 
curtas distâncias, podem ser responsáveis pelos agrupamentos, que se formariam com o transcorrer do tempo no entorno de plantas adultas estabelecidas.

A comparação entre zoocoria-anemocoria utilizando todos os indivíduos da amostra não indicou diferenças significativas quanto ao uso do espaço vertical, ocorrendo médias iguais de alturas para ambos grupos $(\mathrm{P}>0,05)$. Quando considerada apenas a altura máxima das espécies, a média para o grupo anemocórico foi de 19,1 m e para o zoocórico, 15,4 m. A comparação das alturas máximas pelo teste- $t$ de Student indicou diferenças significativas nesses valores $(\mathrm{P}<0,05)$.

A zoocoria possui uma proporção de indivíduos sempre mais elevada que as demais estratégias em todas as classes de altura, fato decorrente de a estratégia ser amplamente difundida entre as espécies amostradas e devido a estas apresentarem as maiores densidades. A anemocoria, além de ser representada por um número menor de espécies, é representada por espécies caracteristicamente de baixas densidades e freqüências, embora com elevadas áreas basais.

Para que árvores de grande porte se desenvolvam até a maturidade em locais sombreados, seus diásporos devem ser maiores, uma adaptação para maior fornecimento de nutrientes às plântulas (Tiffney 1984; Foster \& Janson 1985). Esse aumento no tamanho dos diásporos torna pouco provável a dispersão por outros mecanismos que não vetores bióticos, pois um incremento no peso dos diásporos diminui tanto a eficiência da dispersão balística (Willson 1993), quanto o carreamento pelo vento (Augspurger 1983).

Contudo, muitas espécies emergentes de grande porte contrariam essa constatação, apresentando diásporos suficientemente leves para o transporte pelo vento. Para estas, fatores como a altura da árvore, a velocidade e a direção do vento (Isaac 1930; Horn et al. 2001), a topografia e a vegetação adjacentes (Cremer 1965), e a umidade (McEvoy \& Cox 1987) influenciariam a dispersão. Aparentemente, grande parte dessas variáveis atua uniformizando o investimento na dispersão em todas as direções e para a maior distância possível. Característico de grande parte das espécies dispersas pelo vento, um pequeno investimento de recursos na produção de cada diásporo pode permitir um acréscimo no número destes, favorecendo uma maior área de alcance durante a disseminação. No entanto, caso ocorra pouca variação na direção dos ventos ou estes apresentem baixa velocidade, o padrão de dispersão torna-se pouco aleatório, e os diásporos são carregados a uma pequena parte da área de dispersão possível. Desta forma, a baixa estocasticidade na distribuição de sementes pode ser pouco adaptativa para espécies heliófilas, cujo maior sucesso poderia ser obtido se os propágulos alcançassem uma clareira. Grande parte dos fatores supracitados atua nesse sentido, como por exemplo, o incremento em altura de plantas anemocóricas que torna a copa da árvore emergente, permitindo o acesso desta a ventos inexistentes no dossel contínuo. Árvores altas também propiciam menos obstáculos e maior tempo de permanência no ar aos seus diásporos, permitindo o alcance de maiores distâncias de propagação (Horn et al. 2001). Portanto, alcançar uma altura maior é adaptativo às espécies anemocóricas, pois aumenta a aleatoriedade e o alcance da disseminação.

No caso de $A$. setosa, limitada ao sub-bosque, o tamanho extremamente reduzido dos diásporos (esporos) pode permitir a flutuação e dispersão destes sem a presença de ventos intensos, sendo por vezes chamados de diásporos "sem assistência" (Hughes et al. 1994). Nesse caso, não parece existir pressão seletiva direcionada ao aumento da altura da planta, pois os diásporos são dispersos mesmo no sub-bosque. Alternativamente, a planta produz uma quantidade elevada de esporos para compensar a baixa viabilidade destes, e existem evidências de que a propagação vegetativa contribua com um elevado potencial na produção de novos cáudices (Schmitt \& Windisch 2005).

As espécies zoocóricas possuem, teoricamente, menores chances de disseminar seus diásporos aleatoriamente. Harms et al. (2000) e Jordano \& Godoy (2002) observaram padrões de dispersão por animais com acúmulo de sementes em pontos específicos, enquanto grande parte da área não era atingida pela disseminação, contrastando com os padrões geralmente mais uniformes de espécies anemocóricas. A distribuição das sementes é espacialmente bastante heterogênea devido ao comportamento animal, que pode ocasionar depósitos em sítios preferidos, tais como tocas e ninhos, ou ainda, em trajetos rotineiros de passagem (Schupp et al. 2002). Contudo, para as espécies zoocóricas, a distância alcançada, por exemplo, deve sobrepujar as possíveis vantagens que poderiam ser proporcionadas por uma dispersão espacialmente mais uniforme.

A distribuição espacial agrupada de indivíduos zoocóricos adultos deve ocorrer tanto devido ao acúmulo pontual dos diásporos, quanto à distribuição heterogênea dos fatores limitadores bióticos e abióticos do estabelecimento das plântulas (Harms et al. 2000). 
Aliando esta constatação com a proposição de Schupp et al. (2002), de que a origem e a manutenção da diversidade nas regiões tropicais está ligada à distribuição espacial agrupada, o evento de dispersão passa a ter maior importância.

A partir do presente estudo, as seguintes asserções podem ser efetuadas para a floresta avaliada: 1) a zoocoria é a estratégia de dispersão mais difundida entre espécies, indivíduos e estratos verticais da floresta; 2) os indivíduos adultos anemocóricos são em geral emergentes devido à baixa disponibilidade de ventos nos estratos densos, exceção feita à Alsophila setosa, que possui diásporos suficientemente pequenos para a flutuação em pequenas correntes de ar, sendo a altura elevada adaptativa para as demais espécies do grupo; 3 ) Gymnanthes concolor, autocórica, apresenta pequeno porte e distribuição espacial agrupada provavelmente devido a seu mecanismo primário de dispersão; e 4) o sub-bosque apresenta a maior riqueza específica e densidade de indivíduos zoocóricos, sendo uma fonte de recursos alimentares de grande importância para espécies frugívoras.

\section{Agradecimentos}

À família Canto, pela disponibilização da área de estudo e pelos inúmeros auxílios prestados; ao Programa de Educação Tutorial - PET/SESu/MEC, pela concessão de bolsas aos dois primeiros autores; ao Fundo de Incentivo à Pesquisa - FIPE/CCNE/ UFSM, pelo financiamento de parte deste estudo; aos revisores anônimos, pelas correções e recomendações.

\section{Referências bibliográficas}

Alberti, L.F.; Hirt, J A.N.; Junior, D.B.F.M.; Steckel, M.; Tombini, C.S.; Longhi, S.J. 2000. Aspectos florísticos e síndromes de dispersão das espécies arbóreas do Morro de Santo Antão, Santa Maria-RS. Ciência e Natura 22: 145-160.

APG II - Angiosperm Phylogeny Group. 2003. An update of the Angiosperm Phylogeny Group classification for the orders and families of flowering plants. Botanical Journal of the Linnean Society 141: 399-436.

Augspurger, C.K. 1983. Seed dispersal of the tropical tree, Platypodium elegans, and the escape of its seedlings from fungal pathogens. Journal of Ecology 71: 759-771.

Barroso, G.M.; Morim, M.P.; Peixoto, A.L. \& Ichaso, C.L.F. 1999. Frutos e sementes: morfologia aplicada à sistemática de dicotiledôneas. Viçosa, Editora da Universidade Federal de Viçosa.
Budke, J.C.; Athayde, E.A.; Giehl, E.L.H.; Záchia, R.A. \& Eisinger, S.M. 2005. Composição florística e espectro de dispersão de espécies lenhosas em uma floresta ribeirinha, arroio Passo das Tropas, Santa Maria, RS, Brasil. Iheringia, Série Botânica 32: 17-24.

Budke, J.C.; Giehl, E.L.H.; Athayde, E.A. \& Záchia, R.A. 2004a. Distribuição espacial de Mesadenella cuspidata (Lindl.) Garay (Orchidaceae) em uma floresta ribeirinha em Santa Maria, RS, Brasil. Acta Botanica Brasilica 18(1): 31-35.

Budke, J.C.; Giehl, E.L.H.; Athayde, E.A.; Eisinger, S.M. \& Záchia, R.A. 2004b. Florística e fitossociologia do componente arbóreo de uma floresta ribeirinha, Arroio Passo das Tropas, Santa Maria, RS, Brasil. Acta Botanica Brasilica 18(3): 581-589.

Callegari-Jacques, S.M. 2003. Bioestatística: princípios e aplicações. Porto Alegre, Artmed.

Costa, L.G.S.; Piña-Rodrigues, F.C.M. \& Jesus, R.M. 1992. Grupos ecológicos e a dispersão de sementes arbóreas em um trecho de Floresta Tropical na reserva florestal de Linhares - ES. Revista do Instituto Florestal 4: 303-305.

Cremer, K.W. 1965. Dissemination of seed from Eucaliptus regans. Australian Forestry 30: 33-37.

Foster, R.B.; Arce, J. \& Wachter, T.S. 1986. Dispersal and sequencial plant community en Amazonian Peru Foodplain. Pp. 357-370. In: A. Estrada \& T.H. Fleming (eds.). Frugivores and seed dispersal. Dordrecht, W. Junk.

Foster, S.A. \& Janson, C.H. 1985. The relationship between seed size and establishment conditions in tropical woody plants. Ecology 66(3) 773-780.

Hamilton, W.D. \& May, R.M. 1997. Dispersal in stable habitats. Nature 269: 578-581.

Harms, K.E.; Wright, S.J.; Calderón, O.; Hernández, A. \& Herre, E.A. 2000. Pervasive density-dependent recruitment enhances seedling diversity in a tropical forest. Nature 404: 493-495.

Horn, H.S.; Nathan, R. \& Kaplan, S.R. 2001. Long-distance dispersal of tree seeds by wind. Ecological Research 16: $877-885$

Howe, H.F. \& Smallwood, J. 1982. Ecology of seed dispersal. Annual Review of Ecology and Systematics 13: 201-228.

Howe, H.F. 1986. Seed dispersal by fruit-eating birds and mammals. Pp. 123-189. In: D.R. Murray (ed.). Seed Dispersal. San Diego, Academic Press.

Hughes, L.; Dunlop, M.; French, K.; Leishman, M.R.; Rice, B.; Rodgerson, L. \& Westoby, M. 1994. Predicting dispersal spectra: a minimal set of hypotheses based on plant attributes. Journal of Ecology 82: 933-950.

Isaac, L.A. 1930. Seed flight in the Douglas fir region. Journal of Forestry 28: 492-499.

Janson, C.H. 1983. Adaptation of fruit morphology to dispersal agents in a Neotropical forest. Science 219: 187-189.

Janzen, D.H. 1980. Ecologia vegetal nos trópicos. São Paulo, Editora Pedagógica e Universitária/Editora da Universidade de São Paulo.

Jarenkow, J.A. \& Waechter, J.L. 2001. Composição, estrutura e relações florísticas do componente arbóreo de uma floresta estacional no Rio Grande do Sul, Brasil. Revista Brasileira de Botânica 24(3): 263-272. 
Jordano, P. \& Godoy, J.A. 2002. Frugivore-generated seed shadows: a landscape view of demographic and genetic effects. Pp. 305-321. In: D.J. Levey; W.R. Silva \& M. Galleti (eds.). Seed dispersal and frugivory: ecology, evolution and conservation. New York, CABI Publishing.

Leite, P.F. 2002. Contribuição ao conhecimento fitoecológico do sul do Brasil. Ciência e Ambiente 24: 51-73.

Marchiori, J.N.C. 2000. Dendrologia das angiospermas: das bixáceas às rosáceas. Santa Maria, Editora da Universidade Federal de Santa Maria.

McEvoy, P.B. \& Cox, C.S. 1987. Wind dispersed distance in dimorphic achenes of ragwort, Senecio jacobaea. Ecology 68: 2006-2015.

Morellato, P.C. 1995. Frutos, frugívoros e a dispersão de sementes. Pp.64-76. In: H.F. Leitão Filho \& P.C. Morellato (orgs.). Ecologia e preservação de uma floresta tropical urbana: Reserva de Santa Genebra. Campinas, Editora da Universidade de Campinas.

Moreno, J.A. 1961. Clima do Rio Grande do Sul. Porto Alegre, Secretaria da Agricultura - Div. Terras e Colonização.

Mueller-Dombois, D. \& Ellenberg, H. 1974. Aims and methods of vegetation ecology. New York, Wiley \& Sons.

Nascimento, A.R.T.; Longhi, S.J.; Alvarez Filho, A. \& Gomes, G.S. 2000. Análise da diversidade florística e dos sistemas de dispersão de sementes em um fragmento florestal na região central do Rio Grande do Sul, Brasil. Napaea 12: 49-67.

Oliveira, P.E.A.M. \& Moreira, A.G. 1992. Anemocoria em espécies de cerrado e mata de galeria de Brasília, DF. Revista Brasileira de Botânica 15(2): 163-174.

Schmitt, J.L. \& Windisch, P.G. 2005. Aspectos ecológicos de Alsophilla setosa Kaulf. (Cyatheaceae, Pteridophyta) no Rio Grande do Sul, Brasil. Acta Botanica Brasílica 19(4): 859-865.

Schupp, E.W.; Milleron, T. \& Russo, S. 2002. Dissemination limitation and the origin and maintenance os speciesrich tropical forests. Pp. 19-33. In: D.J. Levey; W.R. Silva \& M. Galleti (eds.). Seed dispersal and frugivory: ecology, evolution and conservation. New York, CABI Publishing.
Sinha, A. \& Davidar, P. 1992. Seed dispersal ecology of a wind dispersed rain forest tree in the western Ghats, India. Biotropica 24(4): 519-526.

Spina, A.P.; Ferreira, W.M \& Leitão Filho, H.F. 2001. Floração, frutificação e síndromes de dispersão de uma comunidade de floresta de brejo na região de Campinas (SP). Acta Botanica Brasilica 15(3): 349-368.

Streck, E.V.; Kämpf, N; Dalmolin, R.S.D.; Klamt, E.; Nascimento, P.C. \& Schneider, P. 2002. Solos do Rio Grande do Sul. Porto Alegre, Editora da Universidade Federal do Rio Grande do Sul.

Tabarelli, M. 1992. Flora arbórea da floresta estacional baixomontana no município de Santa Maria, RS, Brasil. Pp.260-268. In: Anais do II Congresso Nacional Sobre Essências Nativas. Piracicaba, Instituto Florestal.

Teixeira, M.B.; Coura Neto, A.B.; Patore, U. \& Rangel Filho, A.L.R. 1986. Vegetação; as regiões fitoecológicas, sua natureza, seus recursos econômicos; estudo fitogeográfico. Pp. 541-632. In: Levantamento de recursos naturais. Rio de Janeiro, Instituto Brasileiro de Geografia e Estatística, v. 33.

Tiffney, B.H. 1984. Seed size, dispersal syndromes, and the rise of the angiosperms: evidence and hypothesis. Annals of Missouri Botanical Garden 71: 551-576.

Tryon, R.M. \& Tryon, A.F. 1982. Ferns and allied plants with special reference to tropical America. New York, Springer.

van der Pijl, L. 1957. The dispersal of plants by bats (Chiropterochory). Acta Botanica Neerlandica 6: 291-315.

van der Pijl, L. 1982. Principles of dispersal in higher plants. Berlim, Springer-Verlag.

Willson, M.F. 1993. Dispersal mode, seed shadows, and colonization patterns. Vegetatio 107-108: 261-280.

Willson, M.F.; Irvine, A.K. \& Walsh, N.G. 1989. Vertebrate dispersal syndromes in some Australian and New Zealand plant communitites, with geographic comparisons. Biotropica 21: 133-147. 